(C) 1997 International Press

Adv. Theor. Math. Phys. 1 (1997) 127-147

\title{
M-Theory Versus F-Theory Pictures of the Heterotic String
}

\author{
Paul S. Aspinwall \\ Dept. of Physics and Astronomy, \\ Rutgers University, \\ Piscataway, NJ 08855
}

\begin{abstract}
If one begins with the assertion that the type IIA string compactified on a $\mathrm{K} 3$ surface is equivalent to the heterotic string on a four-torus one may try to find a statement about duality in ten dimensions by decompactifying the four-torus. Such a decompactification renders the K3 surface highly singular. The resultant K3 surface may be analyzed in two quite different ways - one of which is natural from the point of view of differential geometry and the other from the point of view of algebraic geometry. We see how the former leads to a "squashed K3 surface" and reproduces the Hořava-Witten picture of the heterotic string in M-theory. The latter produces a "stable degeneration" and is tied more closely to F-theory. We use the relationship between these degenerations to obtain the $\mathrm{M}$-theory picture of a point-like $E_{8}$-instanton directly from the F-theory picture of the same object.
\end{abstract}

\section{Introduction}

As is now well-known, the original multitude of dualities involving various string theories and M-theory are highly interrelated and can be reduced down to some minimal set from which all others may be deduced. For example, Sen [1] showed that the known dualities could be reduced to T-dualities (i.e., dualities between weakly coupled strings that can be deduced from worldsheet methods) and the duality between the type I open string and the $\operatorname{Spin}(32) / \mathbb{Z}_{2}$ heterotic string in ten dimensions. In this approach M-theory 
is defined as the strongly-coupled limit of the type IIA string. We will also adopt this definition of M-theory here even though there has been recent progress in a more intrinsic approach to M-theory [2].

It is, of course, largely a question of taste as to which string duality is to be considered as more fundamental than another. In this paper we will take the duality between the type IIA string on a K3 surface and the heterotic string on a four-torus to be fundamental. One motivation for this is that compactification of closed string theories on complicated objects, such as Calabi-Yau manifolds, is currently better-understood than that for open strings.

If one is to take a six-dimensional pair of string compactifications as a fundamental axiom of string duality then one needs to be able to "decompactify" this dual pair in order to make statements about higher-dimensional theories. It is the geometry of this decompactification process that we wish to discuss in this paper.

The majority of this paper will not be particularly original as most of the results we discuss have appeared in various guises elsewhere, especially in $[3,4]$. Given the large number of dualities at our disposal, any given problem may be solved in a large number of ways. For example, the recent paper [5] covers similar questions to those posed here but from a different viewpoint. The main purpose of this paper will be to emphasize a particularly direct relation between the geometry of F-theory and of M-theory especially where the structure of the $E_{8} \times E_{8}$ heterotic string is apparent. Each picture will turn out to be closely related to a decompactification question.

The moduli space of a type IIA string on a K3 surface and the moduli space of a heterotic string on a four-torus are understood and are exactly the same. This allows us to know exactly which K3 surfaces correspond to exactly which four-torus. See [6] for a review of these facts. We may then ask the question of what exactly happens to the K3 surface as the 1-cycles in the four-torus are taken to be very large. This should tell us how the type IIA string can be dual to the heterotic string in more than 6 dimensions.

An important aspect about this process with respect to the moduli space concerned is that this degeneration consists of going an infinite distance in the moduli space. This is what makes the analysis somewhat subtle.

We will illustrate the two types of limits we wish to consider in the moduli space by examining the well-known moduli space of a two-torus in section 2 . In section 3 we will then squash a $\mathrm{K} 3$ surface as an application of one type of limit. This will reproduce the Horava-Witten picture [7] of the $E_{8} \times E_{8}$ heterotic string on a line segment. We will then use the other kind of limit to produce the stable degeneration picture on F-theory in section 4. Finally in section 5 we use the relationship between the two degenerations to turn an F-theory picture into an M-theory picture. The example studied will be that 
of point-like $E_{8}$-instantons corresponding to 5-branes peeling off the end of the M-theory line segment as in $[8,9]$.

\section{The Two-Torus}

Before we analyze the contortions our K3 surface will undergo in order to decompactify the heterotic four-torus, we will begin with the simplest example of an infinite-distance degeneration. This is that of the two-torus, or elliptic curve.

The usual description of $T^{2}$ is that of the complex $z$-plane divided out by the identifications

$$
z \mapsto z+1, \quad z \mapsto z+\tau,
$$

where $\tau$ is some complex number, $\operatorname{Im}(\tau)>0$. We do not care about the overall size of the torus - just the complex structure. In this case $\tau$ is only defined up to the $\mathrm{SL}(2, \mathbb{Z})$ transformations

$$
\tau \mapsto \frac{a z+b}{c z+d} .
$$

This allows us to restrict $\tau$ to the well-known fundamental region

$$
-\frac{1}{2} \leq \operatorname{Re}(\tau) \leq \frac{1}{2}, \quad|\tau| \geq 1 .
$$

So long as $\tau$ is finite within this region we clearly have a nice smooth torus. In the limit where $\tau$ becomes

$$
\tau_{\infty}=\lim _{\beta \rightarrow \infty} \alpha+i \beta
$$

where $\alpha$ and $\beta$ are real, the torus degenerates. This is the object of interest.

Metrically it is clear what has happened to the torus if we inherit the flat metric of the $z$-plane. One of the 1-cycles has become infinitely larger than the other. Since we didn't fix the overall area of the torus, there are two obvious possibilities. One is that one cycle remains fixed and the other becomes infinite, or that one cycle remains fixed and the other shrinks to zero size.

One answer to the question of what the degenerate torus looks like therefore is that it has become very "skinny" and shrunk itself down to a circle. That is, the dimension has decreased from two real dimensions to one real dimension. Note that the value of $\alpha$ in (2.4) is irrelevant in this limit.

This we take to be the differential geometer's picture of the degenerate torus. If one uses the flat metric on the torus to determine the exact shape of the limit then the above picture is unavoidable. As is well-known however, 
this is not the only way of analyzing the problem. If one asks an algebraic geometer to write down a torus, one might end up with the complex curve

$$
x_{0}^{3}+x_{1}^{3}+x_{2}^{3}-3 \psi x_{0} x_{1} x_{2}=0,
$$

in $\mathbb{P}^{2}$. Now the complex structure is governed by $\psi$ up to the equivalence

$$
\psi \mapsto \omega \psi, \quad \psi \mapsto-\frac{2+\psi}{1-\psi},
$$

where $\omega=\exp (2 \pi i / 3)$.

Now, if $\psi \neq 1$, or equivalent, then this elliptic curve is smooth and is just as good as description of a 2-torus as that given earlier and one may deduce a finite value of $\tau$ corresponding to a given value of $\psi$. It might therefore appear that $\tau=\tau_{\infty}$ corresponds to $\psi=1$. This is not literally true however. When $\psi=1,(2.5)$ factorizes into

$$
\left(x_{0}+x_{1}+x_{1}\right)\left(x_{0}+\omega x_{1}+\omega^{2} x_{1}\right)\left(x_{0}+\omega^{2} x_{1}+\omega x_{1}\right)=0 .
$$

Now each of the three factors in (2.7) is a linear hyperplane in $\mathbb{P}^{2}$ and is therefore a sphere. Each pair of spheres touch each other once. Thus we have a ring of three 2 -spheres.

This picture of the degenerated torus thus corresponds to pinching down three parallel 1-cycles. This is quite different to the differential geometer's answer above. The dimension of the torus hasn't changed at all in the algebraic geometers picture - only a finite number of 1-cycles have been shrunk down. In the differential geometer's picture all the 1-cycles in a given class were shrunk down to zero size.

The degeneration of the torus given by (2.7) is known as a "semi-stable" degeneration. The general idea of a semi-stable degeneration is to write down a fibration

$$
\pi: \mathscr{X} \rightarrow \Delta,
$$

where $\mathscr{X}$ is a smooth algebraic variety of dimension one greater than that required and $\Delta$ is some open subset of the complex $t$-plane. The variety of interest will then be the fibre $\pi^{-1}(t)$ which will be smooth everywhere except at $t=0$ which will correspond to the degenerated space.

That is, a semi-stable degeneration of an elliptic curve corresponds to the possible singular fibres of an elliptic fibration of a complex surface ${ }^{1}$. These were classified by Kodaira and have appeared frequently in the context of F-theory, see, for example, $[3,6]$.

\footnotetext{
${ }^{1}$ One can also demand that the central fibre, $\pi^{-1}(0)$, be a reduced divisor by allowing for suitable base changes. See [10] for more details.
} 
The semi-stable degeneration is not particularly satisfactory for our purposes as the answer is not unique. The idea of a "stable" degeneration is to find the "most generic" semi-stable degeneration and deem it more fundamental than the others. In the case of elliptic curves, the natural degeneration to choose is the one in which precisely one cycle has been shrunk down. See, for example, chapter one of [10] for an explanation of this together with the relevant references.

We have seen in this section that there are two natural models for a degeneration of a torus. To the metrically minded, the torus shrinks itself onto a circle. To the algebraically minded, the degeneration is less severe and consists of just one cycle in the torus shrinking to a point.

\section{M-Theory}

The purpose of this section is to fulfill the promise made in [11] that the tendimensional heterotic string can be understood as M-theory on a squashed K3 surface. This will be consistent with the Hořava-Witten picture [7] in that this squashed $\mathrm{K} 3$ surface will be a finite line segment, $\mathcal{I}$ (i.e., $S^{1} / \mathbb{Z}_{2}$ ). The general principles of this calculation were outlined in [3]. The HořavaWitten picture or squashed K3 surface has also appeared in discussions of $\mathrm{M}($ atrix $)$ theory - see, for example, [12,13].

Our method will be that of the differential geometer who uses a metric on the $\mathrm{K} 3$ surface to analyze the exact way in which it degenerates. We will only consider the $E_{8} \times E_{8}$ heterotic string here. There appears to be no obvious obstacle in applying this technique to the $\operatorname{Spin}(32) / \mathbb{Z}_{2}$ heterotic string although it will be technically harder. If an answer for the $\operatorname{Spin}(32) / \mathbb{Z}_{2}$ heterotic string could be found it would be very interesting given the equivalence of the strongly-coupled $\operatorname{Spin}(32) / \mathbb{Z}_{2}$ heterotic string to the type I string in ten dimensions.

Let us consider the heterotic string on the four-torus. We will decompactify as follows. Firstly switch off all the Wilson lines to give an $E_{8} \times E_{8}$ gauge symmetry. Then set the $B$-field to zero and make the $T^{4}$ be given by a product of four orthogonal circles of radius $\sqrt{\alpha}_{i}$, for $i=1, \ldots, 4$. We may treat all four directions on an equal footing by letting all four radii be equal. We should have in mind then that we are going to ultimately set $\alpha_{1}=\alpha_{2}=\alpha_{3}=\alpha_{4}$. Having said that, in order to present the construction it will be easiest if we allow the notation to distinguish between the various $\alpha_{i}$ 's. We therefore treat the $\alpha_{i}$ 's as independent for most of the time. To decompactify we let $\alpha_{i} \rightarrow \infty$. What does all this do to the K3 surface of the dual type IIA string?

We may answer this question given the explicit form of the map between the heterotic string's four-torus and the type IIA string's K3 surface [14]. 
Most of the manipulations we will perform are explained at length in [6] (in particular, section 5.6) and so we will be brief here. Recall that the moduli space of both the K3 surface and the four-torus in question is given by

$$
\mathscr{M} \cong \mathrm{O}\left(\Gamma_{4,20}\right) \backslash \mathrm{O}(4,20) /(\mathrm{O}(4) \times \mathrm{O}(20))
$$

which may be interpreted as the Grassmannian of space-like 4-planes, $\Pi$, within $\mathbb{R}^{4,20}$, divided by the isometries of the even unimodular lattice $\Gamma_{4,20} \subset$ $\mathbb{R}^{4,20}$.

To achieve the $E_{8} \times E_{8}$ gauge symmetry we decompose

$$
\Gamma_{4,20} \cong \Gamma_{8} \oplus \Gamma_{8} \oplus \Gamma_{4,4},
$$

and impose that $\Pi$ is orthogonal to $\Gamma_{8} \oplus \Gamma_{8}$. This produces two $E_{8}$ quotient singularities in the K3 surface. Let $w^{1}, \ldots, w^{4}$ be four null vectors in $\mathbb{R}^{4,4}$ and let $w_{1}^{*}, \ldots, w_{4}^{*}$ be their duals. Together they generate $\Gamma_{4,4}$. Let $\Pi$ be given by the span of the vectors

$$
w_{i}^{*}+\sum_{j} \psi_{i j} w^{j}
$$

for some matrix $\psi$. One may then show (cf. section 5.6 of [6]) that the desired location in moduli space is given by

$$
\psi=\left(\begin{array}{cccc}
\alpha_{1} & 0 & 0 & 0 \\
0 & \alpha_{2} & 0 & 0 \\
0 & 0 & \alpha_{3} & 0 \\
0 & 0 & 0 & \alpha_{4}
\end{array}\right),
$$

for $\alpha_{i} \rightarrow \infty$.

For the degeneration we wish to perform, we are free to decompose further

$$
\Gamma_{4,4} \cong \Gamma_{2,2} \oplus \Gamma_{2,2},
$$

and assert that $\Pi$ is spanned by two space-like 2-planes, $\Omega$ and $\mho$, each of which is a subspace of $\Gamma_{2,2} \otimes_{\mathbb{Z}} \mathbb{R}$. In terms of the K3 surface this amounts to asserting that we have an algebraic K3 surface whose complex structure is determined by $\Omega$ and whose Kähler form and $B$-field is given by $\mho$. The 24 elements of the cohomology of the K3 surface can be divided into three sets:

1. The 16 classes corresponding to the cycles shrunk to zero size to produce the two $E_{8}$ singularities and hence the $E_{8} \times E_{8}$ gauge symmetry. 
2. The four classes spanning $\Gamma_{2,2}$ giving the "quantum Picard lattice" which is the classical Picard lattice plus $\Gamma_{1,1}$ generated by $H^{0}$ and $H^{4}$. Thus the Picard lattice is $\Gamma_{1,1}$.

3. The remaining transcendental lattice of 2 -cycles given by $\Gamma_{2,2}$.

The fact that the Picard lattice is $\Gamma_{1,1}$ is sufficient to show that the K3 surface is an elliptic fibration with a unique section. The two $E_{8}$ singularities can be

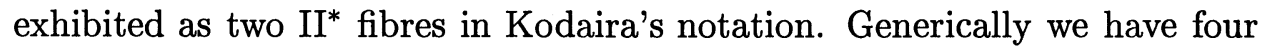
$I_{1}$ fibres elsewhere.

Now we have the upper-left matrix $\left(\begin{array}{cc}\alpha_{1} & 0 \\ 0 & \alpha_{2}\end{array}\right)$ of (3.4) controlling the Kähler form of the K3 surface and the lower-right submatrix $\left(\begin{array}{cc}\alpha_{3} & 0 \\ 0 & \alpha_{4}\end{array}\right)$ controlling the complex structure of the K3 surface. Thus, in the $\alpha_{i} \rightarrow \infty$ limit, both the Kähler form and the complex structure of the K3 surface will degenerate. Note that the choice of $\psi$ in (3.4) sets the $B$-field on the K3 surface to zero.

We will begin by focusing on the Kähler moduli. Let us begin by associating the classes generated by $H^{0}$ and $H^{4}$ to $w_{1}^{*}$ and $w^{1}$. The Picard lattice of the K3 surface is generated by the class of the section and the class of the generic fibre. The section is a rational curve and it thus has self-intersection -2 . Let us call this class $C$ and associate it to the class $w_{2}^{*}-w^{2}$. The generic elliptic fibre we denote $E$ and associate it to $w^{2}$. It can be shown [6] that the Kähler form is then given by

$$
J=\sqrt{\frac{\alpha_{1}}{2 \alpha_{2}}} w_{2}^{*}+\sqrt{\frac{\alpha_{1} \alpha_{2}}{2}} w^{2} .
$$

That is, we have

$$
\begin{aligned}
& \operatorname{Vol}(\mathrm{K} 3)=J . J=\alpha_{1} \\
& \operatorname{Area}(C)=J . C=\sqrt{\frac{\alpha_{1}}{2 \alpha_{2}}}+\sqrt{\frac{\alpha_{1} \alpha_{2}}{2}} \\
& \operatorname{Area}(E)=J . E=\sqrt{\frac{\alpha_{1}}{2 \alpha_{2}}} .
\end{aligned}
$$

The units are those set by the string tension (i.e., the length is set by $\sqrt{\alpha^{\prime}}$ ). In the limit we require we put $\alpha_{2}=\alpha_{1}$ and let $\alpha_{1}$ go to infinity. This produces the following behaviors

$$
\begin{aligned}
\operatorname{Vol}(\mathrm{K} 3) & \sim \alpha_{1} \\
\operatorname{Area}(C) & \sim \alpha_{1} \\
\operatorname{Area}(E) & \sim 1 .
\end{aligned}
$$

That is, the area of the section of the fibration (i.e., the base) grows while the area of the fibre remains fixed. 
Now we consider the complex structure degeneration. We need to understand how both the elliptic fibre and the section deform as $\alpha_{3}$ and $\alpha_{4}$ are taken to infinity. We will need to use our knowledge of the bad fibres in the elliptic fibration to achieve this goal. At two points in the base we have type $\mathrm{II}^{*}$ fibres, blown-down to produce the $E_{8}$ singularities in the $\mathrm{K} 3$ surface, and at four other points we have $\mathrm{I}_{1}$ fibres. The relative locations of these fibres move as the complex structure is varied.

One method, as explained in [3], is to attempt to analyze the metric on the section in terms of deficit angles around the location of the bad fibres. As shown in [15], if the discriminant of the elliptic curve vanishes to order $N$ at a bad fibre, then asymptotically one has a deficit angle of $N \pi / 6$ around a bad fibre. One can then try to model the section in terms of curvature localized around the locations of the bad fibres. This method is somewhat difficult to use to obtain a quantitative picture of the $\mathrm{K} 3$ degeneration in the form we require as it does not lend itself easily to a description in terms of the moduli space we are using.

Instead we will look at the periods of the holomorphic 2-form, $\Omega$. The integral of $\Omega$ over a transcendental 2-cycle (i.e., a 2-cycle that is not homologous to a holomorphic curve) will be generically nonzero. One may fix a hyperkähler (and thus Ricci-flat) metric on the K3 surface while changing the complex structure to turn $\Omega$ into a (suitably normalized) Kähler form. At the same time, if the transcendental cycles are suitably chosen, they will become holomorphic curves. Thus, these periods measure the areas of the transcendental cycles. The suitable transcendental 2-cycles required are the ones whose areas are minimized within a given homology class. These are the "supersymmetric cycles" as discussed in the 3-fold case in [16]. (These object were also discussed in the K3 case in [17].) Knowing these areas will allow us to see how the section and fibre degenerate in the limit we require.

The idea will be to analyze these periods in a way very analogous to the above discussion of the areas of the algebraic cycles. This will allow us to find a symmetry which interchanges various 2-cycles together with the rôles of $\alpha_{1}, \alpha_{2}$, and $\alpha_{3}$.

What are the transcendental 2-cycles in our elliptic fibration? The final answer is fairly simple and has been encountered before in the context of string theory - see, for example, [18]. Technically speaking, for an elliptic fibration $\pi: S \rightarrow B$, the cohomology of $S$ is given by the Leray spectral sequence (see, for example, [19]). Since the fibration has singularities we need to be even more technical and use higher direct image sheaves (see, for example, [20]). Using the fact that the spectral sequence degenerates at $E_{2}$, we have that the second cohomology of our K3 surface is given by the expression

$$
H^{2}(S, \mathbb{Z})=H^{0}\left(B, R^{2} \pi_{*} \mathbb{Z}\right) \oplus H^{1}\left(B, R^{1} \pi_{*} \mathbb{Z}\right) \oplus H^{2}\left(B, \pi_{*} \mathbb{Z}\right) .
$$


Roughly speaking, these objects are not as fearsome as they may first appear. Homologically speaking (i.e., taking the 2-cycle dual to the 2-form), the first term on the right-hand side of (3.9) represents monodromy-invariant 2-cycles in the fibre together with other 2-cycles coming from reducible bad fibres. The only monodromy-invariant 2-cycle in the fibre is, of course, the elliptic fibre itself. The last term represents the section, $C$. All the 2-cycles in the first and final term are algebraic. Any transcendental cycles must be in the term $H^{1}\left(B, R^{1} \pi_{*} \mathbb{Z}\right)$.

Again, roughly speaking, the term $H^{1}\left(B, R^{1} \pi_{*} \mathbb{Z}\right)$ consists of elements dual to 2-cycles built by transporting 1-cycles in the fibre along non-contractable 1-chains in the base. Algebraic 2-cycles in this term correspond to elements of the Mordell-Weil group of the fibration (see, for example, [21]). In our case this group is trivial and so the term $H^{1}\left(B, R^{1} \pi_{*} \mathbb{Z}\right)$ corresponds purely to our desired transcendental 2-cycles.

As mentioned earlier, the transcendental lattice is isomorphic to $\Gamma_{2,2}$. We thus need to build four 2-cycles by transporting 1-cycles in the fibre over 1-chains in the base which generate this lattice.

The first attempt is to take a 1-cycle in the fibre and go around a circle in the base. If this circle can be smoothly contracted then clearly such a constructed 2-cycle will be homologically trivial. Thus the circle must go around locations of more than one bad fibre. In this case however, the 1cycle will usually have monodromy and will not be returned to itself. This method will only work if we can find a collection of bad fibres such that a loop around this collection has no monodromy on 1-cycles in the fibre. To avoid the produced 2-cycle being homologically trivial we also demand that the monodromy individually around each component bad fibre be nontrivial.

Such a path is given by $\gamma_{0}$ in figure 1 . To see this note that a rational elliptic surface may be constructed by taking an elliptic fibration over $\mathbb{P}^{1}$ where the bad fibres are given by a single type $\mathrm{II}^{*}$ fibre and two type $\mathrm{I}_{1}$ fibres. One may then take two rational elliptic surfaces, remove an elliptic curve from each (corresponding to the anticanonical divisor) and glue the two surfaces together along these missing curves to produce a degenerate $\mathrm{K} 3$ surface. This is the stable degeneration of the K3 surface which will be considered in more detail in the next section. For now however we just need to note that $\gamma_{0}$ is the circle that divides the bad fibres into the two sets that can each produce a rational elliptic surface. The fact that these sets of bad fibres can produce a good elliptic surface by themselves must mean that the monodromy around them is trivial.

Either of the 1-cycles that generate $H_{1}$ of the fibre may be transported around $\gamma_{0}$ and each give a 2-torus. Thus we have produced two transcendental 2-tori. Let us call these tori $\mathscr{E}_{1}$ and $\mathscr{E}_{2}$. 


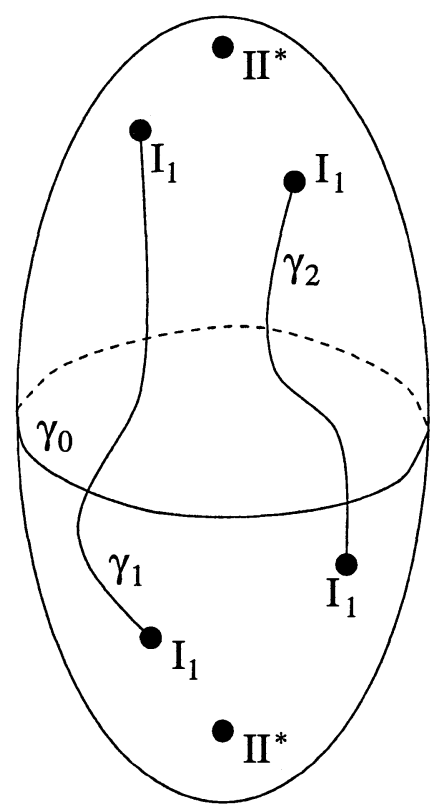

Figure 1: One-chains producing transcendental 2-cycles.

The other two 2-cycles must be built differently. Consider two $\mathrm{I}_{1}$ locations in the base brought together so that they coalesce and produce an $\mathrm{I}_{2}$ fibre. This fibre must be blown-up to produce a smooth elliptic surface. This blow-up produces a new rational curve. Since the total cohomology of the surface cannot have changed during this process, there must have been a transcendental 2-sphere which shrank down to zero size as the two $\mathrm{I}_{1}$ locations approached each other. It is easy to see how to make such a 2-sphere. An $I_{1}$ fibre is exactly the stable degeneration of a 2-torus discussed in section 2 in which one 1-cycle of the fibre shrinks down to zero size. In our case we have two $I_{1}$ fibres in which the same cycle shrinks (otherwise they would not coalesce to form an $\mathrm{I}_{2}$ fibre). The 2-sphere can therefore be built as parallel lines of "latitude" on the 2-sphere given by this 1-cycle in the fibre which shrinks down to zero at the north and south poles. We show this in figure 2.

We may thus associate a transcendental 2-sphere to the path between two $I_{1}$ fibres that would produce a type $I_{2}$ fibre if they were to coalesce. This need not be the case for any pair of type $\mathrm{I}_{1}$ fibres however. Consider the upper pair of $I_{1}$ fibres in figure 1 . We know that these two fibres, together with the upper $\mathrm{II}^{*}$ fibre can produce a rational elliptic surface. The elliptic surface produced by a $\mathrm{II}^{*}$ fibre and a type $\mathrm{I}_{2}$ fibre would have Picard number 11 (one from the section, one from the generic fibre, eight more from the $\mathrm{II}^{*}$ fibre and one more from the $\mathrm{I}_{2}$ fibre). As the rational elliptic surface has 


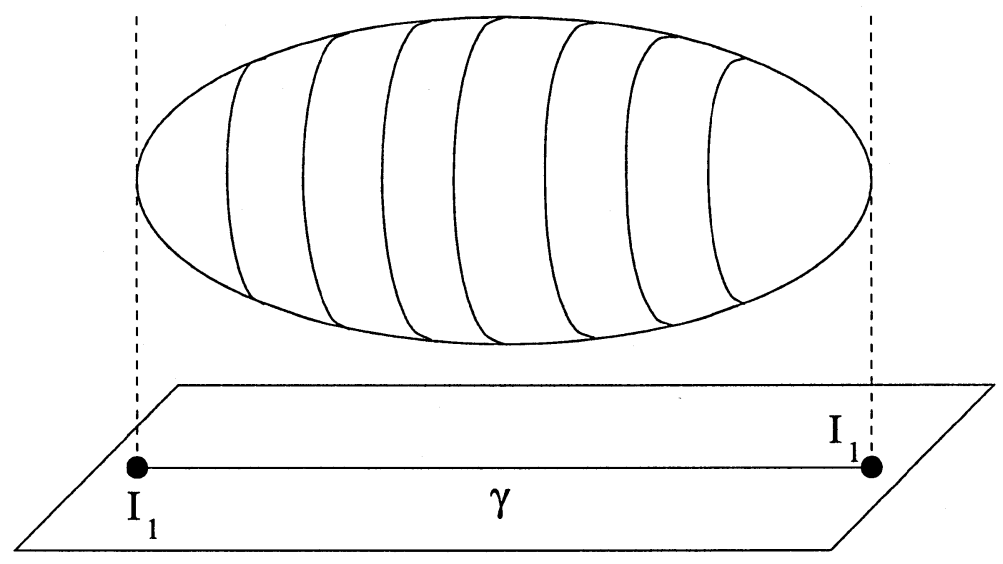

Figure 2: Building a transcendental 2-sphere.

Picard number 10 this is impossible. Each of these type $I_{1}$ fibres contracts a different circle in the fibre. The same is true for the lower pair of $I_{1}$ fibres.

It is not hard to convince oneself that the only way to produce the desired result is to generate two 2-spheres by using the paths shown as $\gamma_{1}$ and $\gamma_{2}$ in figure 1. Let us call these 2-cycles $\mathscr{C}_{1}$ and $\mathscr{C}_{2}$ respectively. One may show that the intersection numbers are given by

$$
\begin{aligned}
& \mathscr{E}_{i} \cdot \mathscr{E}_{j}=0 \\
& \mathscr{E}_{i} \cdot \mathscr{C}_{j}=\delta_{i j} \\
& \mathscr{C}_{i} \cdot \mathscr{C}_{j}=-2 \delta_{i j},
\end{aligned}
$$

thus generating $\Gamma_{2,2}$ as desired.

As we saw earlier, the 2-torus, $E$, together with the 2-sphere, $C$, generate the Picard lattice, $\Gamma_{1,1}$. In addition we now see that the 2-torus, $\mathscr{E}_{1}$, together with the 2-sphere, $\mathscr{C}_{1}$, generate another $\Gamma_{1,1}$ while $\mathscr{E}_{2}$ and $\mathscr{C}_{2}$ generate a third $\Gamma_{1,1}$.

We have now given an explicit construction of the lattice $\Gamma_{4,4}$ in (3.2) as

$$
\Gamma_{4,4}=\Gamma_{1,1} \oplus \Gamma_{1,1} \oplus \Gamma_{1,1} \oplus \Gamma_{1,1} .
$$

The first $\Gamma_{1,1}$ term can be associated to the quantum, $H_{0}$ and $H_{4}$, part of the homology. The second term is the Picard lattice and the third and fourth terms are generated by the transcendental cycles $\left\{\mathscr{C}_{1}, \mathscr{E}_{1}\right\}$ and $\left\{\mathscr{C}_{2}, \mathscr{E}_{2}\right\}$ respectively. The parameters $\alpha_{1}, \alpha_{2}, \alpha_{3}$, and $\alpha_{4}$ can also be associated to each of the four terms in (3.11). Thus by setting these parameters equal, there will be a symmetry of the K3 surface that interchanges these sets. That is, fixing a hyperkähler metric on the $\mathrm{K} 3$ surface, but allowing the complex structure to vary, we may take any of these other three $\Gamma_{1,1}$ lattices to be the Picard lattice. 
Since we set $\alpha_{2}=\alpha_{3}=\alpha_{4}$ any statement about the areas of the 2-cycles $C$ and $E$ must also be true about the 2-cycles $\mathscr{E}_{i}$ and $\mathscr{C}_{i}$. That is from (3.8), in our limit,

$$
\begin{aligned}
\operatorname{Area}\left(\mathscr{E}_{i}\right) & \sim \alpha_{1} \\
\operatorname{Area}\left(\mathscr{E}_{i}\right) & \sim 1,
\end{aligned}
$$

for $i=1$ or 2 .

We now want to know how to relate the areas of the supersymmetric cycles to the lengths of the 1-cycles we have drawn in figure 1 . Since $\pi_{1}(\mathrm{~K} 3)=0$, we have that $H_{2}(\mathrm{~K} 3)=\pi_{2}(\mathrm{~K} 3)$. To build a supersymmetric cycle we can therefore continuously shrink the transcendental cycles we have built above. We will take it as being intuitively clear that the resulting minimal area will be proportional to the product of the shortest possible 1-cycle in the fibre and shortest possible 1-chain in the base in the right class ${ }^{2}$.

That is, up to factors which we are ignoring, the area of a 2-cycle generated by transporting a 1-cycle in the fibre over a one-chain in the base is given by a product of the length of the 1-cycle in the fibre times the length of the one-chain in the base. The only way to achieve all the areas to be the desired size is to make both cycles in the generic fibre of length order one and the chains in the base to satisfy

$$
\begin{aligned}
& \operatorname{Length}\left(\gamma_{0}\right) \sim 1 \\
& \operatorname{Length}\left(\gamma_{1}\right) \sim \alpha_{1} \\
& \operatorname{Length}\left(\gamma_{2}\right) \sim \alpha_{1} .
\end{aligned}
$$

In the above we assume each chain above is some kind of "geodesic" of shortest possible length in its class. The only way to achieve this is to stretch figure 1 into a long vertical sausage of length $\alpha_{1}$.

The picture as $\alpha_{1} \rightarrow \infty$ is therefore of a K3 surface which is stretching out in one direction but remaining fixed in the other three directions. When written as an elliptic fibration, the generic fibre remains fixed in size but the section (or base) elongates in one direction.

Therefore if we take an $E_{8} \times E_{8}$ heterotic string on a four-torus and allow the four-torus to dilate equally in all four directions, the dual type IIA string is compactified on a space which is only large in one dimension. It is interesting to compare this with the calculation in [23] which showed that if the type IIA string's K3 surface is dilated in all four directions equally, then the heterotic four-torus expands in only one direction, leaving a fixed threetorus. This latter correspondence was used to show that M-theory on a K3

\footnotetext{
${ }^{2}$ We are not being particularly rigorous here. One might be able to construct the supersymmetric cycles explicitly along the lines of [22] and then tie it to our construction but we will not attempt this here. I thank D. Morrison for pointing this out to me.
} 
surface is dual to the heterotic string on a three-torus. Our case is somewhat different as we wish to consider the final dual pair in ten dimensions rather than seven.

Let us denote the ten-dimensional heterotic dilaton by $\lambda_{\mathrm{H}, 10}$ and the tendimensional type II dilaton by $\lambda_{\mathrm{II}, 10}$. Similarly, the six-dimensional dilatons are given by $\lambda_{\mathrm{H}, 6}$ and $\lambda_{\mathrm{II}, 6}$, respectively. We also have metrics $g_{\mathrm{H}}$ and $g_{\mathrm{II}}$ by which lengths are measured. We need to be a little careful in the following argument to keep track of the string scale correctly. We use $L$ to denote the natural type IIA scale. That is, our K3 surface above will be of length $L$ in three directions. Now we have

$$
\lambda_{\mathrm{H}, 10}^{2}=\operatorname{Vol}_{\mathrm{H}}\left(T^{4}\right) \lambda_{\mathrm{H}, 6}^{2} \sim \alpha_{1}^{2} \lambda_{\mathrm{H}, 6}^{2},
$$

and

$$
\lambda_{\mathrm{II}, 10}^{2}=\operatorname{Vol}_{\mathrm{II}}(\mathrm{K} 3) \lambda_{\mathrm{II}, 6}^{2} \sim \alpha_{1} L^{3} \lambda_{\mathrm{II}, 6}^{2} .
$$

Heterotic - type II duality however dictates that

$$
\lambda_{\mathrm{H}, 6}=\lambda_{\mathrm{II}, 6}^{-1},
$$

and so

$$
\lambda_{\mathrm{H}, 10}^{2} \sim \frac{\alpha_{1}^{3} L^{3}}{\lambda_{\mathrm{II}, 10}^{2}} .
$$

This shows that as $\alpha_{1} \rightarrow \infty$, the only way that we may maintain a finite coupling for the ten-dimensional heterotic string is to let the type IIA string become infinitely coupled - that is, we require M-theory. Now, as shown in [23], the metric in M-theory is scaled with respect to the type IIA string according to

$$
g_{\mathrm{M}}=g_{\mathrm{II}} \lambda_{\mathrm{II}, 10}^{-\frac{2}{3}} .
$$

Therefore, as $\lambda_{\mathrm{II}, 10} \rightarrow \infty$ M-theory lengths are infinitely shorter than type II lengths. This means that whereas the type IIA string's K3 surface was expanding in one direction, the M-theory K3 surface will actually shrink in the three dimensions. The resulting shape is therefore a line segment which we denote $\mathcal{I}$. This is the "squashed K3 surface" of [11]. To be precise, put

$$
\ell=\alpha_{1} \lambda_{\mathrm{II}, 10}^{-\frac{1}{3}}
$$

as the length of the $\gamma_{1}$ and $\gamma_{2}$ lines in M-theory units, i.e., the length of the squashed K3 surface as measured by M-theory. We also put $L=\lambda_{\mathrm{II}, 10}^{\frac{1}{3}}$. Thus we obtain

$$
\lambda_{\mathrm{H}, 10}^{2} \sim \ell^{3} .
$$


This is in complete agreement with the Hořava-Witten picture [7] which uses quite different logic and describes $\mathcal{I}$ as $S^{1} / \mathbb{Z}_{2}$.

Note that the remnants of the $E_{8}$ structure in the original K3 surface have been squeezed down to points. Thus the geometry of this structure has been lost completely. M-theory itself must therefore just assert that an $E_{8}$ Yang-Mills theory lives at each of the ends of the line segment without giving it a geometrical manifestation. In [7] the existence of the $E_{8}$ 's was shown by anomaly arguments.

\section{F-Theory}

The degeneration of the K3 surface in the previous section was clearly analogous to the degeneration in section 2 of the 2-torus which collapsed onto a circle. That is, every 1-cycle in a given class is shrunk down to zero size resulting in a dimension change from two to one.

In this section we will pursue the algebraic geometer's "stable degeneration" analogous to just shrinking down one cycle of the two-torus. This was proposed in [4] and then explained at length in [24]. We will not repeat that description here but just emphasize certain points. We thus refer the reader to section 3 of [24] for details regarding some of the discussion below.

The first stage of the decompactification in the previous section consisted of stretching the Kähler form so that the section of the K3 surface had an area much greater than the elliptic fibre. We do the same for F-theory. In fact this may be taken as the defining step that takes the type IIA string to F-theory. Again, as in M-theory, one may speculate that F-theory may exist in its own right, rather than just being a limit of string theory. Again we will not pursue such a line of reasoning here (and it seems less likely than for M-theory).

The F-theory statement is therefore an eight-dimensional one in which one asserts that $\mathrm{F}$-theory on a K3 surface is dual to the heterotic string on $T^{2}$ [25]. In contrast to the previous section, this $T^{2}$ will not be decompactified. What we can do however is to ask what happens when this $T^{2}$ becomes very large. This produces the stable degeneration for the F-theory K3 surface.

This may appear to be quite a subtle point as to whether we consider the $T^{2}$ as being decompactified or whether we consider it to be taken to be very large. What is clear however is that it does lead to quite different pictures. For example, the complex structure of this $T^{2}$ remains as a modulus if we merely take the $T^{2}$ to be large. If we decompactify, this modulus is lost as $\mathbb{R}^{2}$ has no complex deformation.

The complex degeneration of the K3 surface we require therefore is one that preserves more geometric structure than the one considered in section 3. By analogy with the 2-torus of section 2 it is clear what we may do. 
We saw in section 3 that the periods associated to the line $\gamma_{0}$ of figure 1 were very small relative to the periods associated to those of $\gamma_{1}$ and $\gamma_{2}$. The M-theory picture of this was to shrink all the cycles in the class of $\gamma_{0}$ down to zero size. This squeezed the 2-sphere in figure 1 down to a line segment. Such a procedure was forced upon us if we wished to respect the Ricci-flat metric - i.e., the hyperkähler structure.
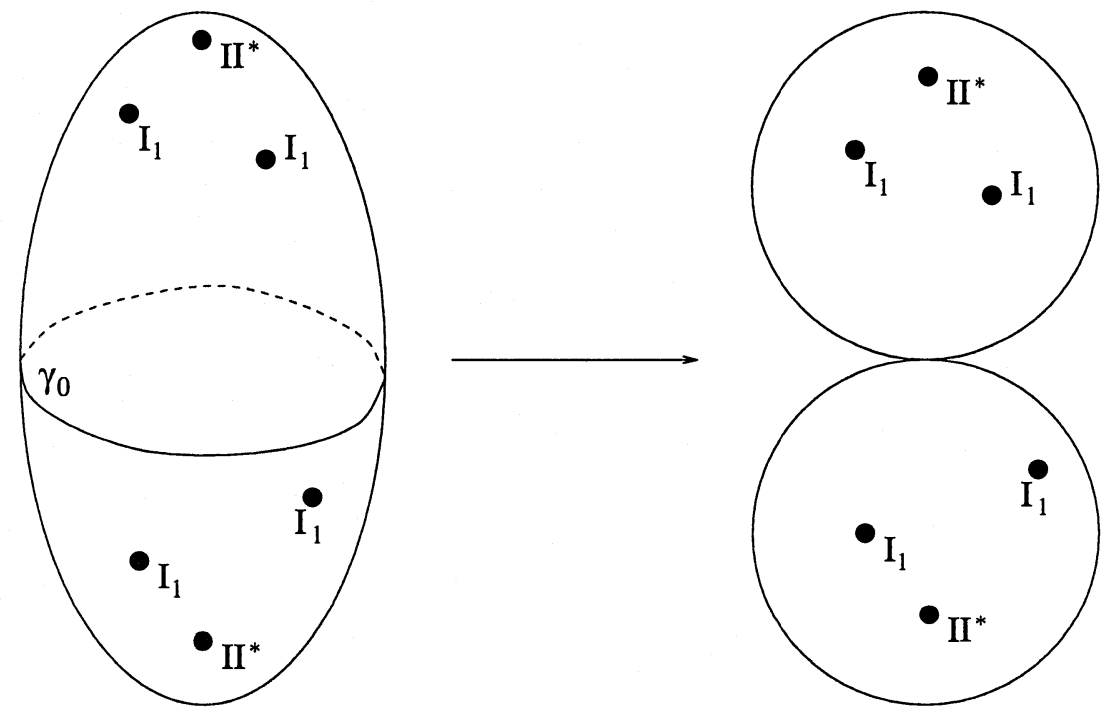

Figure 3: The stable degeneration of an elliptic K3.

If we forget about metrics, we are free to shrink the single cycle $\gamma_{0}$ down to zero size producing figure 3 . This changes the base of the elliptic fibration from a single $\mathbb{P}^{1}$ to two $\mathbb{P}^{1}$ 's intersecting at a point. Therefore, the K3 surface itself degenerates into two rational elliptic surfaces intersecting along an elliptic curve. This latter elliptic curve is the fibre over the point of intersection of the two base $\mathbb{P}^{1}$ 's. This is the stable degeneration of $[4,24]$ and is clearly the analogue of the stable degeneration of the 2-torus in section 2.

It is striking to see just how different the two pictures of the degeneration are. The M-theory degeneration consisted of a line segment with little structure. The F-theory degeneration consists of two rational elliptic surfaces. Again, we should emphasize that the F-theory picture is not a complete decompactification. The complex structure of the $T^{2}$ remains as a modulus. In fact, as explained in $[4,24]$ the modulus of the $T^{2}$ on which the $E_{8} \times E_{8}$ heterotic string is compactified is exactly the modulus of the elliptic curve which is the intersection of the two rational elliptic surfaces on the F-theory side. This elliptic curve, which we will call $E_{*}$, is the fibre over the point of intersection over the two $\mathbb{P}^{1}$ 's in figure 3 . Note also that while the 
$E_{8}$ structures were squeezed out of geometry in M-theory, they are still very much present in the F-theory degeneration in the rational elliptic surfaces.

In order to compactify the $E_{8} \times E_{8}$ heterotic string on $T^{2}$, we need to choose an instanton background for each of the two $E_{8}$ 's on the 2-torus. The moduli space of such objects is (up to some subtleties of $S$-equivalence) the same as the moduli space of semi-stable holomorphic bundles with structure group given by the complex group $E_{8}$. It was explained in [4] (see also the appendix of [26]) that such bundles could be built from $E_{8}$ del Pezzo surfaces and that the moduli space of such del Pezzo surfaces yielded the desired moduli space.

In our case we have rational elliptic surfaces representing the $E_{8}$-bundles rather than del Pezzo surfaces. This is not much of a problem however. A rational elliptic surface can be written as an $E_{8}$ del Pezzo surface blown up at one point. Indeed, the model of the stable degeneration of the K3 surface in question can be flopped so as to replace the rational elliptic surfaces with $E_{8}$ del Pezzo surfaces ${ }^{3}$.

\section{$5 \quad$ M-theory from F-theory}

We have two pictures of the $E_{8} \times E_{8}$ heterotic string on an elliptic curve, $E_{*}$. The F-theory picture, in the large $E_{*}$ limit, is that of the stable degeneration in figure 3 where $E_{*}$ is the fibre (at least as far as complex structure is concerned) over the point of intersection of the two base $\mathbb{P}^{1}$ 's. The M-theory picture is a compactification on $\mathcal{I} \times E_{*}$. It is clear how to produce the Mtheory picture from the limiting F-theory picture - simply squeeze the base $\mathbb{P}^{1}$ 's in figure 3 to produce $\mathcal{I}$. That is, modify figure 3 so that all of the cycles in the class $\gamma_{0}$ shrink to zero size rather that just one.

This should allow the M-theory picture of any F-theory compactification to be constructed. We will illustrate this procedure here by considering point-like $E_{8}$-instantons from the $E_{8} \times E_{8}$ heterotic string compactified on a K3 surface.

In order to compactify the $E_{8} \times E_{8}$ heterotic string on a K3 surface one requires two $E_{8}$-instanton backgrounds on the $\mathrm{K} 3$ surface in order to compactify the gauge degrees of freedom of the heterotic string. In the simplest case, 24 instantons are required to cancel anomalies. These instantons have a moduli space and lead to moduli in the string compactification. These moduli live in hypermultiplets of the uncompactified six-dimensional theory.

\footnotetext{
${ }^{3}$ Actually the construction of [4] can be modified so that it uses the rational elliptic surface directly. In this case the spectral data is built from intersections of elements of the Mordell-Weil group of the rational elliptic curve with $E_{*}$. This construction produces a rather direct picture of the vector bundle data in the stable degeneration and should be studied further.
} 
The branch of the moduli space consisting of such variations is conventionally called the "Higgs" branch. It was shown in $[8,9]$ that if one of these instantons becomes point-like in the K3 surface then new massless moduli may appear leading to a phase transition. These moduli are in tensor multiplets of the six-dimensional field theory and form the "Coulomb branch".

The approach used in $[8,9]$ was based on M-theory compactified on $\mathcal{I} \times \mathrm{K} 3$. This is the picture we wish to "derive". An alternative picture was given in $[3,27]$ based on F-theory. This is the picture we will take as the starting point and which we now review briefly. We refer to [6] for an pedagogical description.

Consider F-theory on a Calabi-Yau threefold, $X$, which is an elliptic fibration over a Hirzebruch surface $\mathbb{F}_{n}$. Let $f$ denote a generic $\mathbb{P}^{1}$-fibre of the Hirzebruch surface and let $C_{0}$ denote the section with self-intersection $-n$. Restricting the elliptic fibration to any $f$ produces a K3 surface. Thus $X$ is also a $\mathrm{K} 3$-fibration over $\mathbb{P}^{1}$.

Unbroken nonabelian gauge groups can be generated by curves of certain fibres within $\mathbb{F}_{n}$ and perturbative gauge symmetries arise from sections of $\mathbb{F}_{n}$ (or multi-sections for higher-level symmetries). We may model all 24 instantons as being small by forcing an $E_{8} \times E_{8}$ unbroken gauge symmetry which is produced by two sections of $\mathrm{II}^{*}$ fibres in $\mathbb{F}_{n}$. When this is done, the geometry allows one to blow-up the base $\mathbb{F}_{n}$ while keeping the elliptic fibration Calabi-Yau. This corresponds to the phase transition produced by the small $E_{8}$-instantons.

This precise geometry of such a transition is shown in figure 4 . In this diagram complex dimensions are drawn as real, i.e., complex curves are drawn as real curves. The horizontal lines and curves denote complex curves of singular fibres of the Kodaira type as indicated. The vertical lines represent $\mathbb{P}^{1}$-fibres, $f$, of the Hirzebruch surface (over which the elliptic fibre is generically smooth). Figure 4 a shows two point-like instantons - one in one $E_{8}$ and one in the other $E_{8}$, each represented in F-theory by collisions of curves of II* fibres with curves of $I_{1}$ as shown. Such a collision can be blown up yielding the transition into the Coulomb branch. This is done for the bottom $E_{8}$-instanton in figure $4 \mathrm{~b}$. This produces a new curve in the base. The effect of this is to replace the $f$-line that had passed through the location of the instanton by two $\mathbb{P}^{1}$ 's intersecting transversely. If we just blow up a little bit, the new $\mathbb{P}^{1}$ that passes through the former location of the instanton will be small while the other $\mathbb{P}^{1}$ will be only slightly smaller than the generic $f$-line.

One may increase the size of the blow-up by changing the relative sizes of the two $\mathbb{P}^{1}$ 's. Note that by homology arguments the total area of the these two $\mathbb{P}^{1}$ 's is fixed and equal to the area of the generic $f$-line. The larger blow-up is shown in figure 4c. We cannot increase the size of the blow-up 


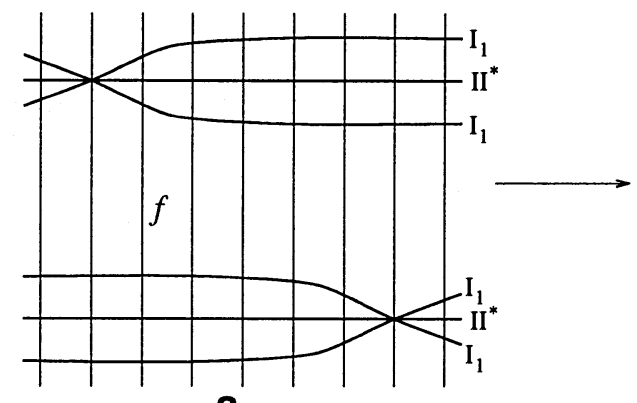

a

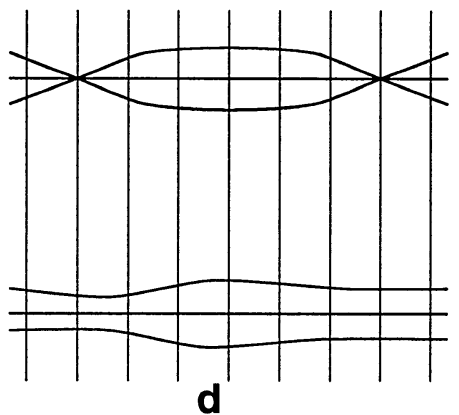

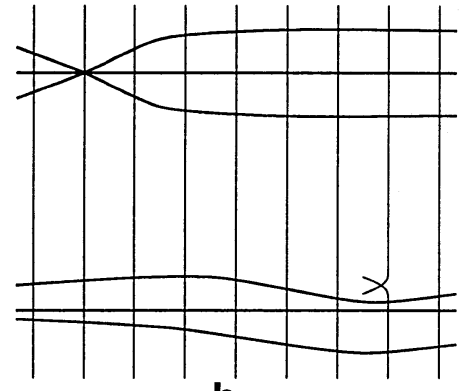

b

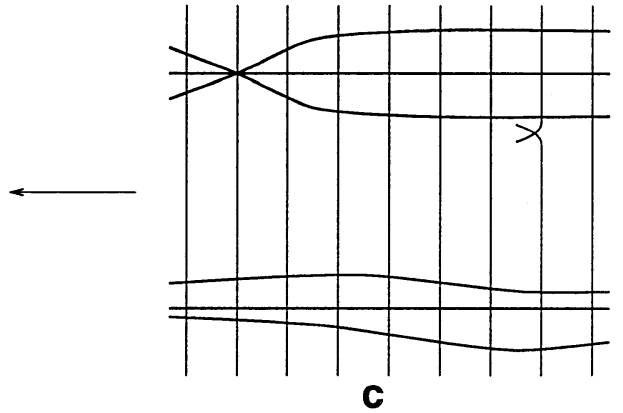

Figure 4: The F-theory picture of moving point-like $E_{8}$-instantons.

indefinitely - eventually the original $\mathbb{P}^{1}$ will shrink down to zero size. This is shown in figure $4 \mathrm{~d}$ and corresponds to the point-like instanton having moved from one $E_{8}$ to the other.

Each $f$-line represents the base of an elliptic K3 surface on which the Ftheory is compactified. We know however that we can turn such a K3 surface into the $\mathrm{M}$-theory picture by squashing this $\mathbb{P}^{1}$ down to a line segment. To be exact, we should first go to the stable degeneration, which picks out a special $T^{2}$ fibre in the elliptic fibration, which we called $E_{*}$. Then we squash the $\mathbb{P}^{1}$ down completely to reproduce M-theory on $\mathcal{I} \times E_{*}$ for each $f$-line.

When we go to the stable degeneration, $E_{*}$ will vary as a move around the base $\mathbb{P}^{1}$ of the Hirzebruch surface. The geometry of the stable degeneration was discussed in [24]. The result is that $E_{*}$ acts as the elliptic fibre of a K3 surface as we move over the base $\mathbb{P}^{1}$. This means that globally the picture becomes M-theory compactified on $\mathcal{I} \times \mathrm{K} 3$.

This is the story for the generic $f$-lines at least. We should also worry about the special fibres in figures $4 \mathrm{~b}$ and $4 \mathrm{c}$ which have become two $\mathbb{P}^{1}$ 's. What happens to these as we go to the M-theory limit?

Actually, squashing these two $\mathbb{P}^{1} \mathrm{~s}$ is exactly the same as squashing the stable degeneration picture of the K3 surface. The equivalence between the 
geometry of stable degenerations and point-like instantons was discussed in [24]. We are thus considering the same degeneration as at the start of this section. That is, each $\mathbb{P}^{1}$ squashes down to a line and then these two lines are joined end-to-end to form a longer line. This longer line will be the same length as the generic I's produced by generic $f$-lines. It is important to note however that the point where these two lines join is significant. As we vary the size of the blow-up, this point will move up and down the resulting line $\mathcal{I}$.

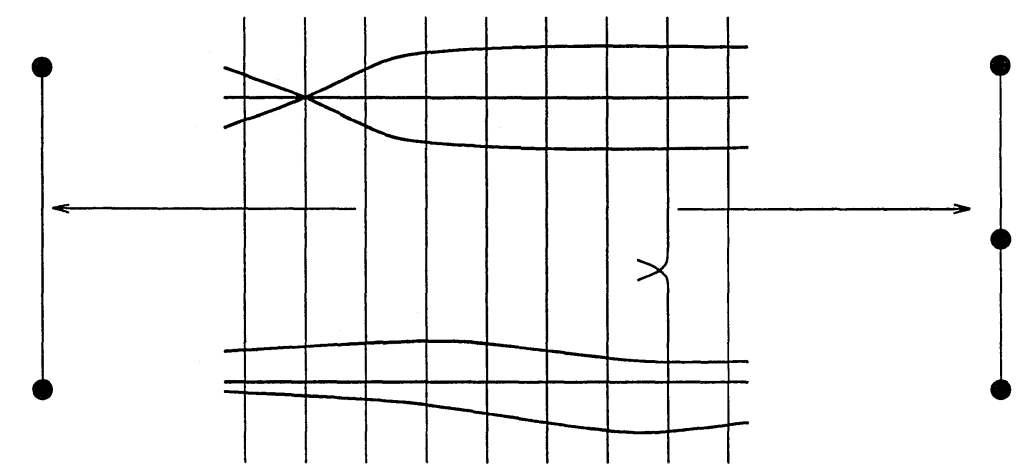

Figure 5: Squashing $f$-lines into $\mathcal{I}$-lines.

This process of moving from F-theory to M-theory is shown in figure 5. The vertical complex $f$-lines in the picture in F-theory are squeezed into real line segments of the form $\mathcal{I}$. The reducible fibres corresponding to blow-ups induced by point-like instantons result in a point being marked within the relevant line segments.

The result then is that figure 4 , in the M-theory limit, will be an $(\mathcal{I} \times$ $E_{*}$ )-bundle over $\mathbb{P}^{1}$, except at the image of the locations of the point-like instantons in the base $\mathbb{P}^{1}$ where the $\mathcal{I}$ will have a special marked point. It is easy to see how to match this up to the picture of $[8,9]$. This special "marked point" on $\mathcal{I}$ is the location of a "5-brane". The transition shown in figure 4 turns into a picture of a 5-brane moving from one end of $\mathcal{I}$ to the other end.

Note that in our description we have only specified the location of the 5 -brane over the base $\mathbb{P}^{1}$ and not as a point in the K3 surface. To fix a point in the $\mathrm{K} 3$ surface we need to further localize the 5-brane to a point in the $E_{*}$-fibre. This degree of freedom is a hypermultiplet degree of freedom and is of the type usually "missed" by F-theory. See [4] for further discussion of these extra moduli in the hypermultiplets.

We see then that the F-theory picture of point-like instantons can be mapped in a very direct fashion to the M-theory picture. 


\section{Acknowledgements}

It is a pleasure to thank O. Aharony, M. Gross, S. Kachru, D. Morrison and E. Silverstein for useful conversations. The author is supported by DOE grant DE-FG02-96ER40959.

\section{References}

[1] A. Sen, Nucl. Phys. Proc. Suppl. 58 (1997) 5.

[2] T. Banks, W. Fischler, S. H. Shenker and L. Susskind, Phys. Rev. D55 (1997) 5112.

[3] D. R. Morrison and C. Vafa, Nucl. Phys. B476 (1996) 437.

[4] R. Friedman, J. Morgan and E. Witten, Comm. Math. Phys. 187 (1997) 679 .

[5] C. V. Johnson, "From M-Theory to F-Theory With Branes", hepth/9706155.

[6] P. S. Aspinwall, "K3 Surfaces and String Duality", in "Fields, Strings and Dualities", C. J. Efthimiou and B. R. Greene, eds, World Scientific 1997.

[7] P. Hořava and E. Witten, Nucl. Phys. B460 (1996) 506.

[8] O. J. Ganor and A. Hanany, Nucl. Phys. B474 (1996) 122.

[9] N. Seiberg and E. Witten, Nucl. Phys. B471 (1996) 121.

[10] R. Friedman and D. R. Morrison, eds, "The Birational Geometry of Degenerations", volume 29 of Progress in Mathematics, Birkhäuser 1983.

[11] P. S. Aspinwall and D. R. Morrison, Phys. Lett. 355B (1995) 141.

[12] M. Berkooz and M. Rozali, "String Dualities From Matrix Theory", hep-th/9705175.

[13] S. Govindarajan, "A Note on M(atrix) Theory in Seven Dimensions with Eight Supercharges", hep-th/9705113, to be published in Phys. Rev. D.

[14] P. S. Aspinwall and D. R. Morrison, "String Theory on K3 Surfaces", in "Mirror Symmetry II", B. Greene and S.-T. Yau, eds, International Press and AMS 1997. 
[15] B. R. Greene, A. Shapere, C. Vafa and S.-T. Yau, Nucl. Phys. B337 (1990) 1.

[16] K. Becker, M. Becker and A. Strominger, Nucl. Phys. B456 (1995) 130.

[17] M. Bershadsky, V. Sadov and C. Vafa, Nucl. Phys. B463 (1996) 420.

[18] A. Klemm et al., Nucl. Phys. B477 (1996) 746.

[19] R. Bott and L. W. Tu, "Differential Forms in Algebraic Topology", Springer-Verlag 1982.

[20] R. Hartshorne, "Algebraic Geometry", Vol. 52 of Graduate Texts in Mathematics, Springer-Verlag 1977.

[21] D. A. Cox and S. Zucker, Invent. Math. 53 (1979) 1.

[22] R. L. Bryant, "Minimal Lagrangian Submanifolds of Kähler-Einstein Manifolds", in "Differential Geometry and Differential Equations", Shanghai 1985, volume 1255 of Lecture Notes in Mathematics, SpringerVerlag 1985.

[23] E. Witten, Nucl. Phys. B443 (1995) 85.

[24] P. S. Aspinwall and D. R. Morrison, "Point-like Instantons on K3 Orbifolds", hep-th/9705104.

[25] C. Vafa, Nucl. Phys. B469 (1996) 403.

[26] M. Bershadsky, A. Johansen, T. Pantev and V. Sadov, "On FourDimensional Compactifications of F-Theory", hep-th/9701165.

[27] D. R. Morrison and C. Vafa, Nucl. Phys. B473 (1996) 74. 